\title{
Adolescência e Saúde: significados atribuídos por adolescentes
}

\author{
Adolescence and Health: meanings assigned by adolescents \\ Adolescencia y Salud: significados asignados por los adolescentes
}

Recebido: 29/01/2021 | Revisado: 02/02/2021 | Aceito: 08/02/2021 | Publicado: 15/02/2021

\author{
Marlon Willian da Silva \\ ORCID: https://orcid.org/0000-0002-4986-119X \\ Universidade Federal de São João del Rei, Brasil \\ E-mail: marlon-willian1993@ hotmail.com \\ Elaine Cristina Dias Franco \\ ORCID: https://orcid.org/0000-0001-8744-7726 \\ Universidade Federal de São João del Rei, Brasil \\ E-mail:elainefranco@ufsj.edu.br \\ Anna Karolina de Oliveira Alfenas Gadelha \\ ORCID: https://orcid.org/0000-0001-8541-9166 \\ Universidade Federal de São João del Rei, Brasil \\ E-mail: karolgadelha6@gmail.com \\ Camila Cristina Costa \\ ORCID: https://orcid.org/0000-0003-3769-8214 \\ Universidade Federal de São João del Rei, Brasil \\ E-mail: camilacosta542@gmail.com \\ Clayton Fernandes de Sousa \\ ORCID: https://orcid.org/0000-0002-3821-1431 \\ Universidade Federal de São João del Rei, Brasil \\ E-mail: claytonfsousa2017@gmail.com
}

\begin{abstract}
Resumo
Objetivo: Analisar, na perspectiva do adolescente, os elementos biopsicossociais que compõem o ser adolescente e sua interface com a saúde. Método: Estudo descritivo, de abordagem qualitativa sustentada no referencial teórico do Interacionismo Simbólico. A coleta ocorreu por meio de entrevista individual, via plataforma virtual, com 12 adolescentes, com idades entre 13 e 16 anos. Para análise dos dados adotou-se a análise de discurso. Resultados: Para os adolescentes, a adolescência é marcada por transformações corporais, fisiológicas e, comportamentais que trazem em certa medida influência para o seu desenvolvimento e saúde. O autocuidado, a prática de atividade física, padrões de sono e alimentação adequados foram considerados como importantes para a saúde na adolescência. Destacam o aumento de responsabilidades e, em algumas circunstâncias, a tomada de decisão relacionada com seus projetos de vida futura. Consideram a adolescência como uma fase difícil, correlacionada com dificuldades de relações interpessoais, principalmente em relação as amizades, baixa estima, aumento de preocupações e alteração de humor associada à manutenção de seu bem-estar psicológico. Considerações Finais: Entender os significados da adolescência e o contexto no qual o adolescente está inserido favorece o desenvolvimento de práticas de educação em saúde potencializadoras da promoção da saúde desse público. Entende-se que essa fase da vida não deva ser vista como um período de transição e sim como parte de um processo de amadurecimento e de intenso aprendizado.
\end{abstract}

Palavras-chave: Adolescente; Desenvolvimento do adolescente; Adolescência; Percepção; Saúde do adolescente.

\begin{abstract}
Objective: To analyze, from the adolescent's perspective, the biopsychosocial elements that make up the adolescent and their interface with health. Method: Descriptive study, with a qualitative approach based on the theoretical framework of Symbolic Interactionism. The collection took place through individual interviews, via virtual platform, with 12 adolescents, aged between 13 and 16 years. For data analysis, the discourse analysis was adopted. Results: For adolescents, adolescence is marked by bodily, physiological and behavioral transformations that, to a certain extent, influence their development and health. Self-care, physical activity, sleep patterns and adequate nutrition were considered important for adolescent health. They highlight the increase in responsibilities and, in some circumstances, decision-making related to their future life projects. They consider adolescence as a difficult phase, correlated with difficulties in interpersonal relationships, especially in relation to friendships, low esteem, increased concerns and changes in mood associated with maintaining their psychological well-being. Final Considerations: Understanding the meanings of adolescence and the context in which the adolescent is inserted favors the development of health education practices that enhance the health promotion of this public. It is understood that this phase of life should not be seen as a period of transition, but as part of a process of maturation and intense learning.
\end{abstract}

Keywords: Teenager; Adolescent development; Adolescence; Perception; Adolescent health. 


\begin{abstract}
Resumen
Objetivo: Analizar, desde la perspectiva del adolescente, los elementos biopsicosociales que componen al adolescente y su relación con la salud. Método: Estudio descriptivo, con enfoque cualitativo basado en el marco teórico del Interaccionismo Simbólico. La recolección se realizó a través de entrevistas individuales, vía plataforma virtual, con 12 adolescentes, con edades entre 13 y 16 años. Para el análisis de datos se adoptó el análisis del discurso. Resultados: Para los adolescentes, la adolescencia está marcada por transformaciones corporales, fisiológicas y conductuales que, en cierta medida, influyen en su desarrollo y salud. El autocuidado, la actividad física, los patrones de sueño y una nutrición adecuada se consideraron importantes para la salud de los adolescentes. Destacan el aumento de responsabilidades y, en algunas circunstancias, la toma de decisiones relacionadas con sus proyectos de vida futuros. Consideran la adolescencia como una etapa difícil, correlacionada con dificultades en las relaciones interpersonales, especialmente en relación a las amistades, baja estima, aumento de preocupaciones y cambios de humor asociados al mantenimiento de su bienestar psicológico. Consideraciones finales: Comprender los significados de la adolescencia y el contexto en el que se inserta el adolescente favorece el desarrollo de prácticas de educación en salud que potencien la promoción de la salud de este público. Se entiende que esta etapa de la vida no debe verse como un período de transición, sino como parte de un proceso de maduración y aprendizaje intenso.
\end{abstract}

Palabras clave: Adolescente; Desarrollo de los adolescentes; Adolescencia; Percepción; Salud del adolescente.

\title{
1. Introdução
}

Considerada pela Organização Mundial de Saúde (OMS), como um fenômeno contemporâneo, a adolescência é uma fase do desenvolvimento humano que apresenta como delimitação tanto critérios cronológicos e físicos, como também sociais e culturais (OMS, 2009). A OMS considera o limite entre os 10 e 19 anos para a adolescência, sendo este dividido em duas etapas: (I) pré-adolescência (de 10 a 14 anos) marcada pelas primeiras mudanças físicas, hormonais, maturação cognitiva e sexual e; (II) adolescência propriamente dita (de 15 a 19 anos) que compreende a integração social onde há uma necessidade de enfrentamento de suas limitações e desenvolvimento saudável (OMS, 2009). No Brasil, o Estatuto da Criança e do Adolescente define o adolescente como um indivíduo em condição peculiar de desenvolvimento e com idade entre 12 e 18 anos (Brasil,1990).

Pode-se inferir que a adolescência é um período de desenvolvimento humano caracterizado por transições biopsicossociais. É uma fase de desconstrução da infância, colaborando para maturação, independência, reorganização emocional, pertencimento a grupos sociais e transformações físicas (Brasil 2017). No campo psicossocial são comuns alterações nas relações escolares, a experimentação intensa de diversos sentimentos, a busca pela autonomia e independência familiar, bem como a predileção em experimentar novos comportamentos e vivências acompanhadas com frequência de contestação de ideias e conceitos preestabelecidos. Já no campo biológico percebe-se, de forma evidente, alterações físicas e hormonais, dentre outras (Costa et al, 2019; Malta et al, 2014). Dessa forma, a saúde dos adolescentes pode ser diretamente afetada, interferindo em seu desenvolvimento na fase adulta.

Nas últimas décadas, os adolescentes constituem um grupo prioritário para a promoção da saúde considerando que seus comportamentos e vivências, associados ao contexto histórico, social e cultural em que vivem, podem desencadear situações desfavoráveis para a saúde desse público (Costa et al, 2019). Diversos países, com apoio da Organização Mundial da Saúde (OMS), desenvolveram ao longo das últimas décadas um sistema de vigilância baseado em inquéritos (Health Behaviour in School-aged Children - HSBC; Youth Risk Behavior Surveillance System - YRBSS, KIDSCREEN versões 52, 27 e 10, dentre outros) relacionados à saúde de escolares, os quais incluem informações sobre a saúde física, emocional e psicológica, influência da escola, família e pais e de fatores socioeconômicos (Oliveira, Campos, Andreazzi, \& Malta, 2017; Malta et al, 2014).

As intervenções com adolescentes têm revelado que ao abordá-los há a necessidade de constituir caminhos que sejam sustentados no conceito ampliado de saúde, em seus determinantes como condições "sine qua non" para a qualidade de vida e para a experimentação/fortalecimento da promoção da saúde, considerando dimensões relacionadas á saúde física e psicológica, nível de independência, relações sociais e o meio ambiente. Há a necessidade de intervenções multidisciplinar e 
interdisciplinares que envolvam os diversos aspectos que compõem a adolescência e o "ser adolescente", a exemplo do papel social, incluindo a retomada ao trabalho; a capacidade funcional; a imagem corporal; o lazer e; as relações interpessoais que no âmbito da adolescência são acrescidas as mudanças fisiológicas, psicológicas, cognitivas e sociais que compõem essa fase do ciclo vital (Farias, Loch, Lima, Sales, \& Ferreira, 2017).

Diante desse contexto diverso que representa a adolescência e de suas diferentes interfaces, busca-se com este estudo analisar, na perspectiva do adolescente, os elementos biopsicossociais que compõem o ser adolescente.

\section{Metodologia}

Estudo descritivo de abordagem qualitativa sustentado no referencial teórico do Interacionismo Simbólico, que considera o significado como uma linha de sustentação para a compreensão do comportamento humano, das interações e dos processos que compõem o cotidiano dos indivíduos (Carvalho, Borges \& Rêgo, 2010; Bueno, Alves, \& Vasques, 2017). No contexto da adolescência, onde o cotidiano mostra-se disforme e em constante (re)construção, o Interacionismo Simbólico mostra-se como uma abordagem alinhada para a análise dos processos de (re)socialização e também para o estudo de mudanças de opiniões, comportamentos, expectativas e exigências sociais que compõem o "ser adolescente".

O protocolo de pesquisa cadastrado com número do CAEE: 26421719.4.0000.5545, aprovado sob parecer n. 4.305.238 emitido em 28 de setembro de 2020, foi apreciado pelo Comitê de Ética à luz da Resolução 510/2016 que estabelece as diretrizes para pesquisa com Seres Humanos no campo das ciências sociais.

Inicialmente foram identificados 51 possíveis participantes selecionados de forma intencional, uma vez que deviam ter idade entre 13 e 16 anos e serem acompanhados pelo Programa de Residência Multiprofissional em Saúde do Adolescente implantado em um município do oeste mineiro. Todos foram contactados por telefone e foram convidados a participar do estudo. Após o primeiro contato foram obtidos 12 retornos favoráveis à participação com a anuência do responsável legal e o assentimento do adolescente. Os motivos para a não adesão dos demais participantes foram a indisponibilidade de tempo; a ausência de recurso tecnológico para participar da entrevista virtual e; a preferência por contato pessoal e este não ser possível à época da coleta devido as medidas restritivas de contato durante a pandemia da COVID-19.

As entrevistas ocorreram no período de outubro a dezembro de 2020 em horário previamente agendado com o adolescente, via plataforma Google Meet, com duração média de 15 minutos. Mediante a autorização do adolescente e de seu responsável legal, houve a gravação do áudio da entrevista para auxílio da transcrição das narrativas e posterior análise. Para garantia do sigilo e anonimato todos os participantes receberam a codificação alfabética- numérica representada pela letra $\mathrm{A}$ seguida do número de sequência da entrevista (A1, A2, A3 ...).

Diante do uso de plataforma virtual para a entrevista adotou-se como conduta a presença de dois pesquisadores, sendo um moderador e um observador. O moderador teve como atribuições: (I) explicar ao adolescente a dinâmica da entrevista; (II) estabelecer a identificação do participante para facilitar o registro e transcrição do áudio; (III) estimular a participação do adolescente a partir da adoção de estratégias de estimulação de produção de narrativas com o uso de expressões como "me fale mais sobre isso"; "sinta-se à vontade para falar"; "conte-mais um pouco sobre isso", dentre outros. Quanto ao observador, este ficou responsável pelas anotações no diário de campo relacionadas aos momentos em que ocorreram mudanças na entonação da voz do participante como, por exemplo, sussurros, aumento de volume, risos, pausas, bem como as possíveis interferências relacionadas à tecnologia. Em pesquisas qualitativas a forma como o partipante produz sua narrativa pode revelar sua maior ou menor implicação com seu discurso e a temática que compõe sua fala.

Para acessar a sala virtual da entrevista, ambas as partes (pesquisadores e adolescentes), estavam em local privativo e silencioso, de forma que não ocorressem interrupções e fossem preservados o anonimato e sigilo dos participantes. $\mathrm{Na}$ entrevista, o adolescente foi conduzido a narrar suas percepções acerca: (I) da adolescência, como uma fase do ciclo da vida; 
(II) do fato de ser e se perceber neste momento como adolescente; (III) da relação que estabelecem entre saúde e adolescência e como percebem o seu autocuidado. Os dados empíricos foram analisados considerando os referenciais teóricos da Análise de Discurso (Fairclough, 2016).

\section{Resultados e Discussão}

As aproximações e análises das práticas discursivas dos/das adolescentes participantes do estudo trouxeram à luz duas categorias temáticas que versam sobre: I- Ser adolescente: o intermezzo entre os limites de tempo, os marcos corporais, os sentimentos e a experiência do vivido; II- Saúde e Adolescência: (im)possibilidades de articulação.

\section{I- Ser adolescente: o intermezzo entre os limites de tempo, os marcos corporais, os sentimentos e a experiência do vivido}

Ao serem impelidos a narrar sobre suas percepções acerca do que é "SER ADOLESCENTE" os participantes delimitam a adolescência considerando os marcos de faixa etária e as mudanças corporais, conforme trechos a seguir:

No meu caso eu notei dos 12 para 13 anos uma diferença... Foi aí que eu percebi.... Eu fui crescendo, os pelos desenvolvendo e eu fui notando uma mudança no meu corpo, a voz também. (A6)

Para mim acho que começa aos 13, 12 anos. Foi quando começou a minha adolescência. (A10)

Acho que começa com 11 para 12 anos, eu acho...foi quando virei mocinha. (Al1)

Acho que de uns 13 anos para cima né... você começa a reparar que está aparecendo pelos, barba, bigode, a voz engrossa um pouco, seu corpo começa a crescer né. (A7)

$\mathrm{Na}$ tentativa de definir a "adolescência" os atores sociais partem de suas experiências pessoais, marcadas pelo uso de expressões como "No meu caso", "Para mim" revelando uma implicação com o que desejam dizer e ao mesmo tempo uma tentativa de tornar suas narrativas consistentes. Para eles, a adolescência tem delimitação de idade inicial quando dizem "começa aos 13,12 anos", "começa com 11 para 12 anos" e também é marcada por transformações corporais quando dizem, por exemplo, "fui notando uma mudança no meu corpo", "seu corpo começa a crescer".

Diante dos significados sociais já estabelecidos para a adolescência, é comum que nas situações que busquem definíla ocorra a menção aos limites etários que são considerados como balizas para a continuidade das discussões e para as conformações dos discursos daqueles que ali estão, conforme observa-se nas narrativas dos adolescentes que buscam estabelecer uma idade inicial para adolescência. Os adolescentes usam limites de idades que estão definidos na literatura e nas legislações associados às alterações corporais que podem ser percebidas por aqueles que estão no seu entorno e são reconhecidas socialmente como marcas de transição do corpo infantil para a adolescência e posteriormente para a idade adulta.

$\mathrm{Na}$ busca por delimitar e compreender de forma detalhada as diferentes fases que compõem o ciclo da vida, especialistas definem a adolescência a partir de um recorte de faixa etária compreendida entre os 10 e 19 anos pela OMS ou entre os 12 e 18 anos, no Brasil, quando a referência é o Estatuto da Criança e do Adolescente (Brasil, 1990; OMS, 2009). E também associam a essa faixa etária às mudanças biopsicossociais que compõem o cotidiano dos indivíduos que estão neste ciclo da vida (Davim, Germano, Menezes, \& Carlos, 2009).

$\mathrm{Na}$ continuidade do processo de construção de suas narrativas com o intento de definir a adolescência, os adolescentes a relacionam como um período singular da vida, uma transição entre o "SER CRIANÇA" e "SER ADULTO", marcada por descobertas de novos pensamentos, comportamentos, bem como maturidade associada a aquisição de responsabilidades, autonomia e liberdade, conforme os relatos a seguir: 
Ser adolescente? É não ser criança e nem adulto tá no meio dos dois. É um processo assim né, de sair de criança para ser adulto, amadurecer. É uma fase de amadurecimento. (A7)

Eu percebo a adolescência pelo comportamento, né, que vai mudando ao longo do tempo. Eu acho que mudei muito quando passei a ser adolescente. Eu brincava muito, muito mesmo de boneca e hoje em dia eu não gosto (A8)

Ser uma pessoa mais responsável. (A5)

Agora que a gente está descobrindo as coisas né, vamos dizer assim... descobrindo as coisas... Por que eu acho que é aí que você começa a entender mais, começa a sair da fase de criança, ser inocente e começa a enxergar outras coisas, como elas realmente são... eu me vejo tomando as minhas decisões, seguindo o que eu tenho que seguir, claro, com direcionamentos, mas, tomando as minhas próprias decisões. (A11)

Observa-se que os adolescentes se sentem envolvidos quanto optam por narrarem suas experiências demarcadas com o uso de pronome pessoal "EU" e verbos no indicativo "percebo", "acho". Para os participantes a adolescência mostra-se como um período singular e ao mesmo tempo intenso uma vez que é marcado por um "abandono" do corpo e comportamentos infantis em prol de um novo status biopsicossocial. Essas transformações são tidas como indispensáveis na vida dos indivíduos, configurando a adolescência como uma fase crítica permeada por momentos de definições de identidade sexual, profissional, de valores e sujeita a inúmeros desafios, incluindo adaptação às mudanças fisiológicas e anatômicas. As diversas modificações físicas, cerebrais, endócrinas, emocionais, sociais e sexuais, ocorrem de forma simultânea, com modificações estruturais, físicas, mentais e emocionais que vão dar origem a novos comportamentos e emoções sentidas pelo adolescente (Davim, Germano, Menezes, \& Carlos, 2009). Há desse modo, conforme as narrativas dos participantes, o reforço à percepção identificada em um estudo realizado com estudantes de São Paulo, no qual os autores destacam que a aquisição de novas funções sociais e responsabilidades promovem o distanciamento do adolescente das nuances da infância (Brêtas, Moraes, Freitas, Goellner, \& Godoi, 2020).

Entretanto, diante da diversidade de situações que envolvem à adolescência, os participantes também a consideram como uma fase difícil relacionada com a ruptura de laços de amizade, baixa autoestima, incertezas, mudanças de humor e solidão, conforme trechos a seguir:

Aaah. é uma fase difícil né! Por que é uma fase em que a gente perde muitos amigos e ah as vezes a gente fica com a autoestima baixa...é uma fase muito difícil! Ah por que muitas vezes os amigos trocam...assim, não quer continuar a amizade né! (A10)

Ser adolescente não é fácil né, é muitas coisas que acontece com a gente, às vezes é muita responsabilidade, na escola também a gente não dá conta de certas coisas e a gente fica preocupado...são muitas coisas a fazer, muitas coisas a pensar e isso só vai acrescentando mais, né, porque a vida adulta também é a mesma coisa, é a continuação disso. (A4)

É uma fase muito complicada...é uma fase onde mesmo que a gente queira a todo momento pular ela, é necessária por que essa transição as vezes assusta... por causa dos hormônios, dos sentimentos, dessas coisas que vem demais, vem à tona muito forte, que a gente não consegue controlar, mas sem ela também a gente assustaria mais, em questão das responsabilidades da vida adulta. (A3)

Eu acho confuso...éh ser adolescente é confuso. Porquê... por muitos motivos... por exemplo, eu sou velha para algumas coisas e nova para outras coisas. Por isso, nova pra fazer algumas coisas e velha pra fazer outras coisas. Eu 
às vezes não posso me, tipo, impor o que eu penso, mas aí se eu não me imponho aí eles falam 'mas você devia ter falado", mais para esse sentido.(A9)

Ser adolescente é difícil né? Vivendo várias emoções, às vezes a gente se estressa do nada. (A12)

Eu me vejo ...uma pessoa fechada do mundo, assim, das pessoas. Porque eu me isolo muito. (A8)

Nos discursos dos adolescentes, observa-se uma modalidade subjetiva, caracterizada pelo uso de verbo no presente do indicativo "acho", do pronome pessoal "EU" e também do uso da expressão "a gente" para caracterizar o EU, traduzindo o quanto os atores sociais estão implicados na busca por uma resposta que melhor represente o Ser Adolescente e as dificuldades que representam essa fase. Essa construção discursiva coloca em relevo uma relação que perpassa não apenas o texto, mas também sinaliza para a identificação dos sujeitos com sua prática social (Fairclough, 2016), ainda que, em alguns momentos, as pausas e verbalizações de dificuldades e os desejos manifestos de "pular" esse período da vida se tornem presentes.

As responsabilidades inerentes ao processo de transição da infância para a adolescência e desta última como um passo para a vida adulta tornam o processo mais difícil para adolescentes. Esse fato é observado quando eles destacam que há um encadeamento crescente de responsabilidades com o avanço dos anos que compõem a adolescência com uso de expressões "são muitas coisas a fazer", "só vai acrescentando", "responsabilidades da vida adulta", dentre outras. A busca pela independência e autonomia configura-se como uma condição sine qua non para o pleno desenvolvimento da identidade do adolescente, no entanto, isso não acontece de forma simples. São comuns os momentos de conflitos, dúvidas e de sentimentos de confusão para o adolescente, observado quando dizem "ser adolescente é confuso", "eu sou velha para algumas coisas e nova para outras coisas", "essa transição as vezes assusta". Nesse cenário são frequentes os sentimentos de ansiedade e frustração que, muitas vezes, geram expectativas e inseguranças quanto ao futuro e como se portar (Faria \& Ponciano, 2018).

A adolescência é permeada pelos participantes por dificuldades que estão relacionadas com a interação com seus pares, representada pelos laços de amizade e a manutenção destes. Nesse ínterim, diante das numerosas mudanças pessoais, sociais e emocionais que permeiam a adolescência pode-se inferir que a qualidade das relações estabelecidas entre cada adolescente e o seu grupo de pares está associada à manutenção de seu bem-estar psicológico, com sua capacidade de integração social e para superar com os desafios de ser adolescente já que contribuem para a interiorização de valores como a confiança, a lealdade, a reciprocidade e a responsabilidade (Carvalho \& Novo, 2013; Carvalho, et al. 2017). Estudos que tratam da adolescência e suas características multidimensionais no desenvolvimento do sujeito a revelam como uma fase caracterizada por frequentes mudanças socioemocionais que são acompanhadas de um amadurecimento progressivo da competência emocional e de autopecepção favorecendo o melhor controle das emoções nas relações com seus pares, na sociedade e também consigo mesmo (Ponciano \& Féres-Carneiro, 2014; 2017; Santrock, 2014).

\section{II- Saúde e Adolescência: (im) possibilidades de articulação}

Na perspectiva dos adolescentes, a saúde relaciona com atendimento a condições básicas para a manutenção da vida como a alimentação e o sono que são, por vezes, associados ás práticas de atividade física, bem-estar emocional e social.

Saúde, é você dormir bem, se alimentar bem, fazer exercícios físicos. Como comer de tudo, carne, arroz, feijão, verdura, frutas, tendo limites para refrigerantes, doces... (A6)

Saúde é uma coisa que a gente sempre tem que ter...tipo assim, por exemplo, a saúde emocional, a gente sempre tem que tentar ter uma saúde emocional boa, tentar controlar nossa mente...e a saúde do corpo, tem que também tentar se alimentar bem, a gente tem que ter uma saúde boa, e pra isso a gente tem que se alimentar bem, fazer academia. 
(A12)

Saúde ... eu acho que bem estar, estar se sentindo bem é saúde... Quando tá tudo feitinho, quando os trabalhos estão entregues, quando não tem muita coisa pra preocupar...eu me sinto bem. (A9)

Saúde pra mim é a gente poder cuidar da gente mesmo. A gente poder ter amor, amor a saúde que a gente tem, porque se a gente não se alimentar bem, se a gente não fizer uma atividade física, se a gente não cuidar da gente mesmo... a nossa saúde só vai ficando prejudicada e isso pode trazer sérios problemas pra todos nós. (A4)

Há um movimento de exposição de perspectivas e entendimentos particulares dos atores sociais, que tem como referências as práticas saudáveis como promotoras de saúde que constituem as políticas de assistência à saúde e são reafirmadas em diversos espaços de convivência dos adolescentes, a exemplo da escola, academias, livros escolares e também redes sociais como instagran, facebook, dentre outras. Entretanto, observa-se que no contraponto do conceito ampliado de saúde, o adolescente A7 vislumbra a saúde dentro de uma perspectiva biomédica associada à ausência de doença, revelando que a intencionalidade de um conceito ampliado de saúde não compõe seu cotidiano:

Para mim, sei lá, saúde é... sei lá, não tá doente, né? Não tá morrendo de doença aí, acho que pra mim isso é saúde. (A7)

A hegemonia do modelo assistência de saúde centrado na doença encontra reforço em diversas práticas sociais reforçando a ideia social de que estar saudável representa não ter doenças. Desse modo, observa-se que mesmo em face de legislações e políticas públicas que valorizem o conceito amplidado de saúde há em determinadas circunstâncias a redução da saúde aos aspectos biológicos em detrimento dos elementos psicossociais que compõem a ser humano e seu cotidiano.

Para além do conceito de saúde, os participantes foram estimulados a estabelecer uma correlação entre saúde e adolescência. Ao responderem ao estímulo, os participantes A6 e A11 trouxeram à luz seus cotidianos sustentados em práticas esportivas, alimentação e espiritualidade:

Saúde e adolescência é fazer isso... ter a limitação na alimentação, fazer exercícios físicos, é isso, é você movimentar. (A6)

Saúde e adolescência é fazer esporte, é uma coisa que adolescente gosta ne?! exercício físico, alimentação e vida espiritual também. (A11)

E para a adolescente A4 saúde e adolescência podem ser expressas por meio do autocuidado representado em seu discurso pela expressão "cuidar de si mesmo".

Eu acho que saúde e adolescência pode estar ligada a cuidar de si mesmo e a pensar também, a raciocinar, porque se a gente for parar pra pensar se a gente não raciocinar nos nossos hábitos, na nossa saúde ou na nossa adolescência, traz consequências, como, ou uma doença na saúde ou um problema na adolescência, a gente sempre tem que tá por dentro das coisas da adolescência e das coisas da saúde. Então a saúde com a adolescência são complementares, porque uma ajuda a outra, eu penso assim, porque a saúde influencia muito na adolescência, por o ser humano estar crescendo, o metabolismo dele funcionar melhor, a saúde influencia muito na adolescência, porque se a nossa saúde 
não estiver boa a gente não vai ter um crescimento bom, a gente não vai ter uns ossos fortes e tudo mais. (A4)

Quando indagados sobre os cuidados com a saúde, os adolescentes revelam um deslocamento entre comportamentos pautados em práticas saudáveis e situações de risco para a saúde, conforme trechos a seguir:

Para cuidar da minha saúde eu faço academia, mas eu me alimento bem errado...eu acho que é preciso uma boa alimentação e de atividade física, mas eu faço atividade física e me alimento errado...comer ruim, um monte besteira, tomar refrigerante todo final de semana, sair comendo pizza, cachorro-quente, hambúrguer, essas coisas...comer muito sal... (A1)

Eu cuido da minha saúde fazendo exercício físico, eu jogo bola, tendo dormir bem, cerca de 7 horas por dia. (A6)

Para cuidar da minha saúde eu me alimento bem. Tem algumas coisas que eu não gosto, aí eu não como não. Mas como frutas, verduras, uma alimentação variada. Como também doces e bebo refrigerantes, que não fazem bem para a saúde. Aí cuido me alimentando da maneira melhor que tem, tentando evitar o que não faz bem...também jogo bola.

Vixe, eи acho que eu não cuido da minha saúde, mas tudo bem, eu poderia melhorar nesse aspecto.... tem dia que eu não quero comida, tipo, almoço, mas ficar só na bolacha, mas tem dias que eu tô super...ai quero comer arroz, feijão e todas essas coisas saudáveis e tem dias que eu só quero ficar no meu quarto comendo besteira...(A9)

A gente, acaba que, fica mexendo mais no celular, quem trabalha igual eu, a gente só vai trabalhar, chega come besteira, então acaba que não fica tão bom. (A12)

Em seus relatos acerca de "Como cuidam de sua saúde", os atores sociais adotam em seus discursos o uso das estruturas linguísticas "eu cuido", "eu acho", "minha saúde”, dentre outras. O uso dessas expressões marca a natureza subjetiva de suas afirmações e denota uma condição de implicação com suas escolhas para o autocuidado, conferindo às práticas discursivas uma modalidade subjetiva (Fairclough, 2016). Observa-se que há um deslocamento entre práticas saudáveis para a saúde e situações de risco nos discursos dos participantes. Esse fato pode estar relacionado às novas influências ambientais vivenciadas por eles, que têm como pano de fundo a aceitação de seus pares, as mudanças nos padrões de comunicação e das rotinas. Esse conjunto de fatores favorece aos adolescentes a adoção de hábitos, nem sempre muito saudáveis, como alimentarse fora de casa, recorrer a comidas de fácil acesso, além do uso prolongado de tecnologias, a exemplo do celular (Senna \& Dessen, 2015).

\section{Considerações Finais}

Por meio de suas práticas discursivas, os adolescentes, atores sociais deste estudo, trouxeram à luz suas experiências singulares frente ao período da adolescência permitindo a identificação das dimensões biopsicossociais que compõem essa fase do ciclo vital. Considerando os marcos cronológicos, os adolescentes percebem essa fase com início entre 11 e 13 anos de idade.

Para os adolescentes seus cotidianos são marcados por transformações corporais, fisiológicas e, comportamentais que trazem em certa medida influência para o seu desenvolvimento e saúde. Há também como característica dessa fase o aumento de responsabilidades e, em algumas circunstâncias, a tomada de decisão relacionada com seus projetos de vida futura. A adolescencia é considerada para alguns adolescentes como uma fase difícil, correlacionada com dificuldades de relações 
interpessoais, principalmente em relação as amizades, baixa estima, aumento de preocupações, alteração de humor associada à manutenção de seu bem-estar psicológico.

As questões relacionadas a percepção e articulação de adolescência e saúde se deram pela indagação sobre o que era saúde para eles. Houve neste momento o destaque para a prática de atividades físicas, padrões de sono e alimentação adequados. Para alguns adolescentes a saúde emocional também é uma preocupação e o autocuidado foi visto como prática de saúde importante, mas em algumas circunstâncias negligenciada por eles, ainda que saibam quais são os comportamentos de risco e os saudáveis para a manutenção da saúde.

Diante dos desfechos que compõem o corpus deste estudo pode-se inferir que seu objetivo primário de analisar, na perspectiva do adolescente, os elementos biopsicossociais que compõem o ser adolescente foi atingido. Conclui-se que a percepção do adolescente perante a fase da adolescência permite uma compreensão dos componentes biopsicossociais que o cerca. É possível identificar a compreensão dos adolescentes sobre o conceito de saúde e suas implicações em seus cotidianos, visto que a consciência de vulnerabilidade em saúde parte das situações de risco que os mesmos se colocam. Os resultados apresentados neste estudo poderão corroborar para o planejamento e execução de práticas de educação em saúde que atendam às demandas singulares que compõem o universo de "ser adolescente" promovendo ao longo do tempo o fortalecimento da promoção da saúde. Entende-se que essa fase da vida não deva ser vista como um período de transição e sim como parte de um processo de amadurecimento e de intenso aprendizado. A tendência de ver a adolescência como "um período de transição" tem favorecido o esquecimento das necessidades desta população e, para que as políticas sociais e de saúde possam de fato dar a esse público a integralidade que lhe é devida, é necessário "dar voz a esse público", entender o que representa "Ser adolescente". A limitação deste estudo está relacionada às dificuldades de acesso aos adolescentes provocada pelas medidas de distanciamento social durante a Pandemia da COVID-19.

\section{Referências}

Brasil. (2017). Ministério da Saúde. Secretaria de Atenção à Saúde. Departamento de Ações Programáticas e Estratégicas, B. (2017). Proteger e cuidar da saúde de adolescentes na atenção básica. Ministério da Saúde.

Brasil. (1999). Estatuto da Criança e do Adolescente, Câmara dos Deputados, Lei no 8.069, de 13 de julho de 1990. DOU de 16/07/1990 - ECA. Brasília, DF. p. 36.

Brêtas, J. R. S., Moraes, S. P.de, Freitas, M. J. D. de, Goellner, M. B., \& Godoi, A. M. L. de. (2020) Corpo do adolescente: subsídios para intervenção. Research, Society and Development, 9 (11), e67991110082. http://dx.doi.org/10.33448/rsd-v9i11.10082

Bueno, T., Alves, M., \& Vasques, F. F. (2017). Interacionismo Simbólico como ferramenta teórica e metodológica para o estudo no ciberespaço. Razón y Palabra, 21 (96), 456-475. https://www.redalyc.org/articulo.oa?id=1995/199551160024

Carvalho, R. G., Fernandes, E., Câmara, J., Gonçalves, J. A., Rosário, J., Freitas, S., \& Carvalho, S. (2017). Relações de amizade e autoconceito na adolescência: um estudo exploratório em contexto escolar. Estudos de Psicologia (Campinas), 34(3), 379-388. https://dx.doi.org/10.1590/198202752017000300006

Carvalho, V. D. de, Borges, L. O., \& Rêgo, D. P. do. (2010). Interacionismo simbólico: origens, pressupostos e contribuições aos estudos em Psicologia Social. Psicologia: Ciência e Profissão, 30(1), 146-161. https://dx.doi.org/10.1590/S1414-98932010000100011

Carvalho, R. G., \& Novo, R. F. (2013). Características da personalidade e relacionamento interpessoal na adolescência. Avaliação Psicológica, 12(1), 27-36.

Costa, C. C., Franco, E. C. D., Santos, T. M., Silveira, E. A. A. da, Carvalho, M. S., \& Resende, M. A. A. (2019). Perfil biopsicossocial de crianças e adolescentes institucionalizados. Revista Eletrônica Acervo Saúde, 11(17), e1671. https://doi.org/10.25248/reas.e1671.2019

Davim, R. M. B., Germano, R. M., Menezes, R. V. M., \& Carlos, D.J.D. (2009). Adolescente / adolescência: revisão teórica sobre uma fase crítica da vida. Rev Rene, 10 (2), 131-140. https://www.redalyc.org/articulo.oa?id=3240/324027966015

Fairclough N. Discurso e Mudança Social. (2016). Brasília: Universidade de Brasília.

Faria, A. P. S., \& Ponciano, E. L. T. (2018). Conquistas e fracassos: os pais como base segura para a experiência emocional na adolescência. Pensando familias, 22(1), 87-103. http://pepsic.bvsalud.org/scielo.php?script=sci_arttext\&pid=S1679-494X2018000100008\&lng=pt\&tlng=pt.

Farias, J. C. J. de, Loch, M. R., Lima, A. J. de, Sales, J. M., \& Ferreira, F. E. L. de L. (2017). Reprodutibilidade, consistência interna e validade de construto do KIDSCREEN-27 em adolescentes brasileiros. Cadernos de Saúde Pública,33(9), e00131116. Epub September 28, 2017.https://doi.org/10.1590/0102$311 \times 00131116$ 
Research, Society and Development, v. 10, n. 2, e27510212482, 2021

(CC BY 4.0) | ISSN 2525-3409 | DOI: http://dx.doi.org/10.33448/rsd-v10i2.12482

Malta, D. C., Andreazzi, M. A. R. de, Oliveira-Campos, M., Andrade, S. S. C. A., Sá, N. N. B., Moura, L., Dias, A. J. R., Crespo, C. D., \& Silva Júnior, J. B. (2014). Tendência dos fatores de risco e proteção de doenças crônicas não transmissíveis em adolescentes, Pesquisa Nacional de Saúde do Escolar (PeNSE 2009 e 2012). Revista Brasileira de Epidemiologia, 17(Suppl. 1), 77-91. https://doi.org/10.1590/1809-4503201400050007

Oliveira, M. M. de, Campos, M. O., Andreazzi, M. A. R. de, \& Malta, D. C. (2017). Características da Pesquisa Nacional de Saúde do Escolar PeNSE. Epidemiologia e Serviços de Saúde, 26(3), 605-616. https://doi.org/10.5123/s1679-49742017000300017

Organização Mundial de Saúde. (2009). Child and adolescent health and development. Genebra: OMS. http://www.who.int/child-adolescent-health/

Ponciano, E. L. T., \& Féres-Carneiro, T. (2017). Conjugalidade, parentalidade e separação: repercussões no relacionamento pais e filhos(as). Psicologia Em Estudo, 22(2), 277-287. https://doi.org/10.4025/psicolestud.v22i2.32808

Ponciano, E. L. T., \& Féres-Carneiro, T. (2014). Relação Pais-Filhos na Transição para a Vida Adulta, Autonomia e Relativização da Hierarquia. Psicologia: Reflexão e Crítica, 27(2), 388-397. Recuperado em 10 janeiro, 2021 de https://doi.org/10.1590/1678-7153.201427220

Santrock, J. W.(2014). Adolescência (14th ed.). Rio de Janeiro: AMGH Editora.

Senna, S. R. C. M., \& Dessen, M. A. (2015). Reflexões sobre a saúde do adolescente brasileiro. Psicologia, Saúde \& Doenças, 16(2), 217229. https://dx.doi.org/10.15309/15psd160208 